\title{
FRACTURE OF THE BRONCHUS *
}

\author{
BY \\ J. LLOYD GRIFFITH \\ From Frenchay Park Hospital, Bristol
}

Traumatic rupture of the bronchus is an unusual condition, and is sufficiently rare to justify the reporting of one further case.

The case presented shows many unisual features. The injury sustained may be termed simple fracture rather than rupture, or laceration of the bronchus.

The common complications of this type of injury did not arise. Pneumothorax, bleeding into the pleural cavity, surgical emphysema, and collapse of the lung did not occur, presumably because the mucosa and membranous wall of the bronchus remained intact.

\section{CASE History}

The patient was a woman aged 37 years. She was involved in a motor-car accident in July, 1947, and appears to have been thrown against the steeringwheel of the car she was driving.

She was unconscious for about half an hour, and on admission to hospital was found to have a fracture of the maxilla with two teeth missing, a fractured ankle, and severe bruising of the anterior chest wall.

The day following she was very short of breath and had marked stridor. Her chest felt very sore. She developed a persistent dry cough but produced no blood or sputum.

Radiographs of the chest on the day following the accident showed fractures of the first, second, third, and fourth ribs on the left side with no displacement; there was no air or fluid in the pleural cavities, and the lung fields appeared normal. No foreign body was visible.

After a few days the dyspnoea and stridor disappeared. On becoming ambulatory dyspnoea returned, wheezing became a marked feature, and was associated with a dry cough.

The patient was referred to me in September, 1947, as a case of laryngeal obstruction. She was admitted to the Thoracic Unit, Frenchay Park Hospital, Bristol, in October, 1947.

I have outlined the history. She stated that she had not suffered from any chest trouble before the accident. Since she had been able to get about the dyspnoea had increased, and had prevented her from returning to work.

*A paper read before the Society of Thoracic Surgeons of Great Britain and Ireland at Victoria Park Hospital, London, in the autumn of 1948.
Examination.-Marked inspiratory and expiratory wheeze was present. This was greatly accentuated by slight exercise, which caused obvious dyspnoea. Her colour was good. She coughed incessantly, but produced no sputum. The trachea and larynx were normal to feel.

On auscultation a loud inspiratory and expiratory rhonchus was audible over the mid-zone of the chest on the left side; it was best heard posteriorly. No other chest abnormality was found on clinical examination.

Investigations.-Indirect laryngoscopy showed the vocal cords to be normal, and they moved normally and equally.

Radiographs of the chest disclosed fractures of the first, second, third, and fourth ribs on the left side, with no displacement, and callus formation present. There was no fluid and no air in the pleural cavity, nor any pleural thickening present. The lung fields, heart, and mediastinum appeared normal.

Radiographs were then taken in inspiration and expiration; in the expiratory phase obstructive emphysema of the left lung was evident with shift of the mediastinum to the right, suggesting that there was an obstruction in the left main bronchus causing delay in the escape of air from the left lung.

At bronchoscopy the cords appeared normal and moved equally; the trachea was normal, as was also the carina. On the right side no abnormality was seen, but on the left side, about half-way between the carina and the upper lobe orifice, there was a stricture, the normal lumen being reduced to about half a centimetre. Mobility and motility were normal. The stricture was lined with normal mucosa and there was no ulceration. It was crescentic in contour. The stricture was dilated and enlarged readily, with no feeling of resistance. The stricture appeared unlike an ordinary fibrous one; it seemed elastic in nature, as on withdrawing the largest bougie from it the constriction promptly returned to its former size. The narrowing was certainly not malignant in nature ; it gave the impression that it consisted of mucosa and soft tissue only without any cartilaginous support. In other words, simple fracture of the bronchial wall had occurred with displacement, the narrowing being due to overriding and mal-alignment of the two ends.

Tomograms were taken to try to demonstrate the region of the narrowing in the left main bronchus. Unfortunately satisfactory films were not obtained. I did not feel justified in trying to outline the region by bronchography, as I feared that the oil might 

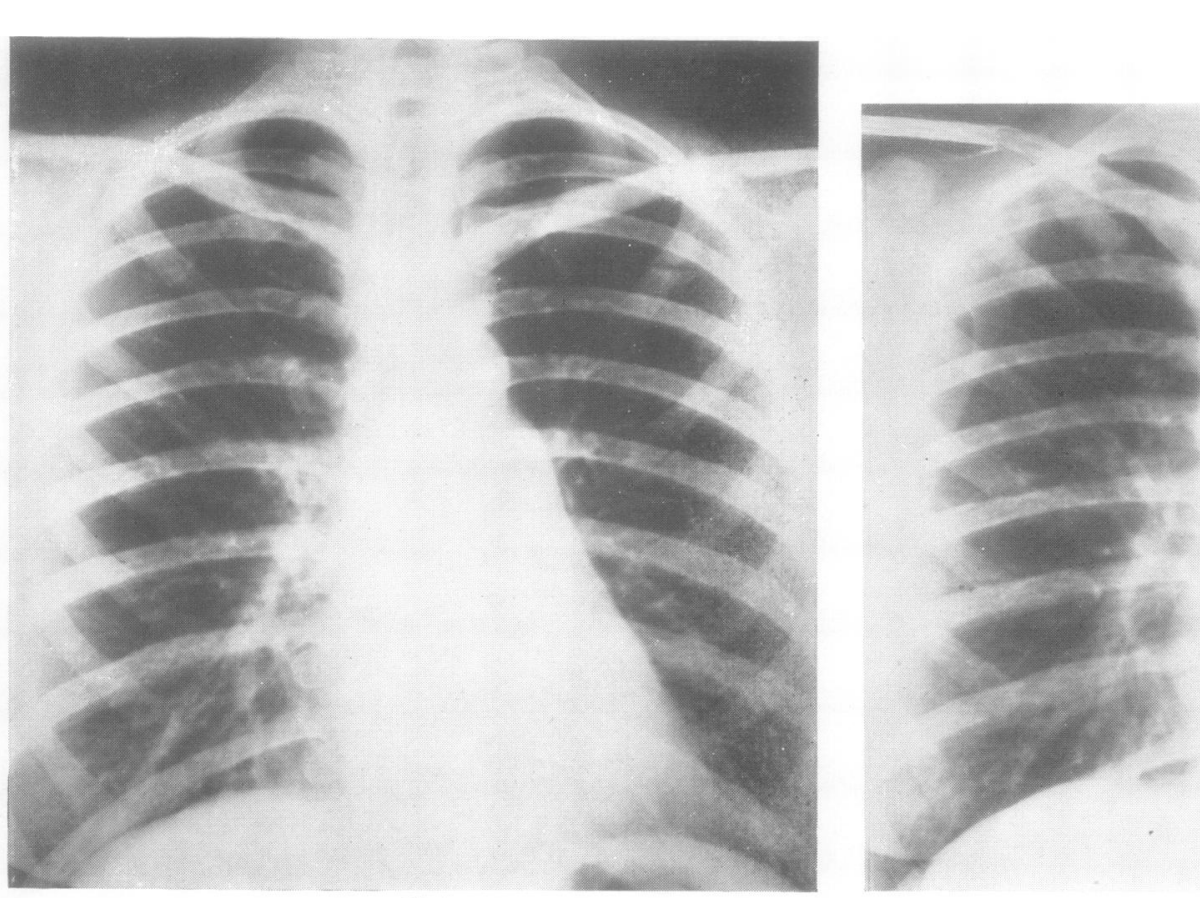

(1)
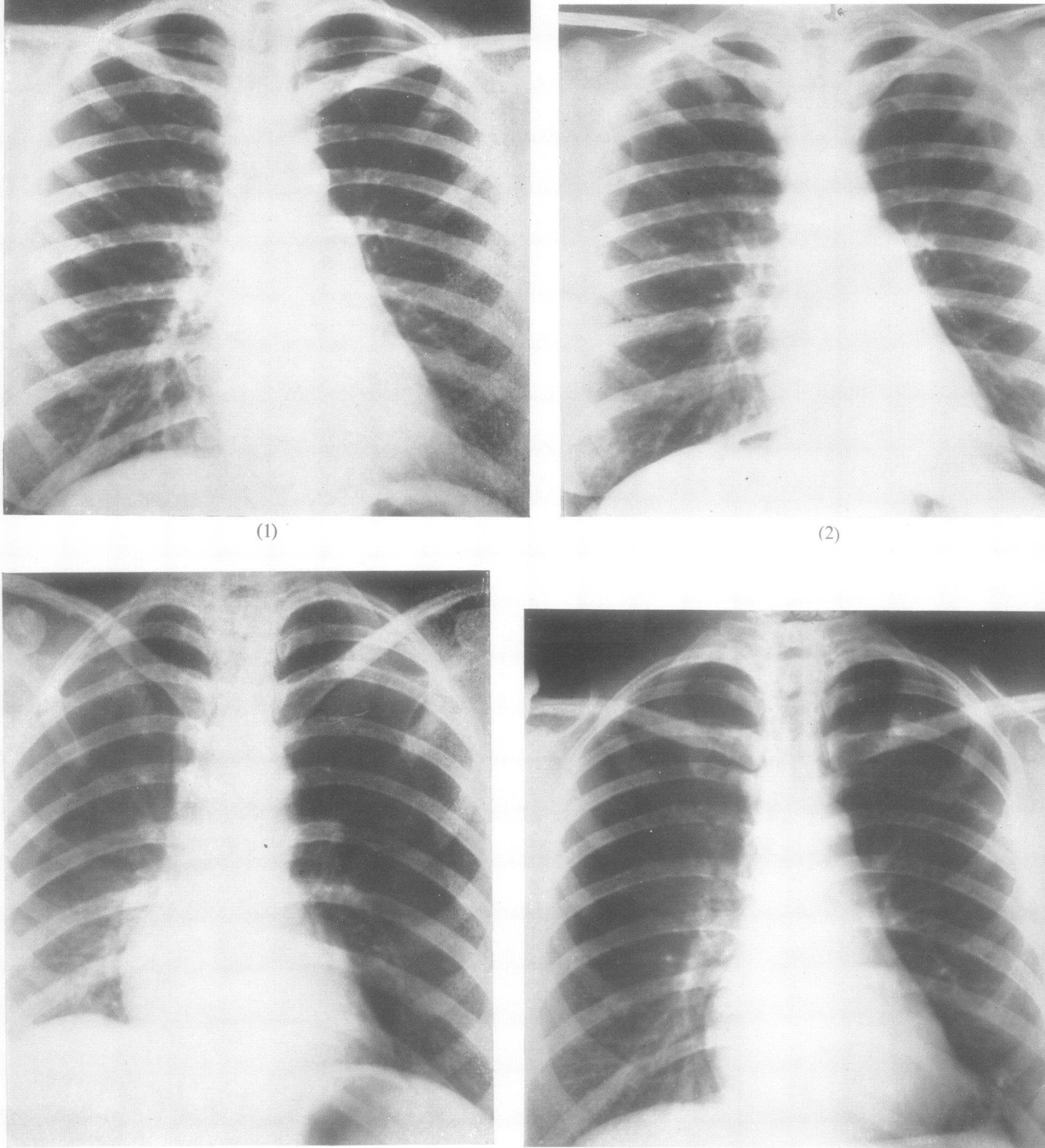

(3)

$(4)$

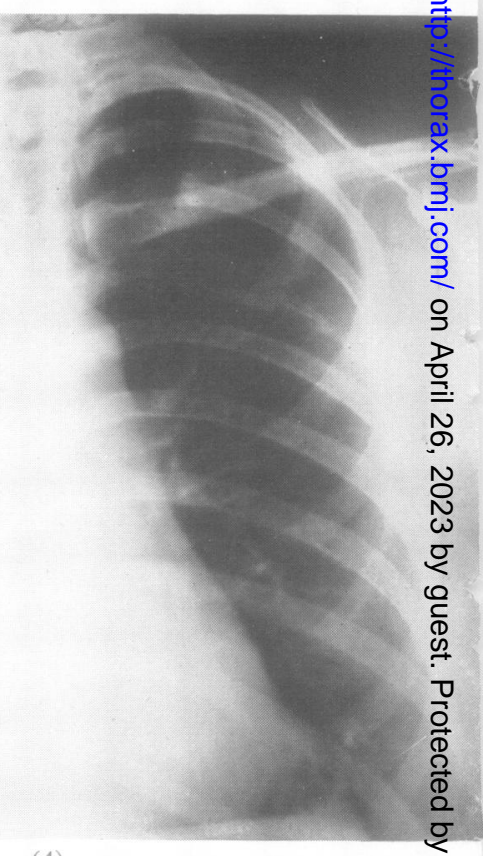

FiG. 1.--Postero-anterior view of chest (Sept.10,1947) shortly after admission, showing no definite abnormality in lung fields, but fract of first, second, third, and fourth ribs with no displacement. Fig. 2.-Position of lungs in inspiration (Dec. 4). FIG. 3.-Position of lungs in expiration (Dec. 4), showing shift of mediastinum to the right with obstructive emphysema of left lung. FiG. $\underset{\stackrel{P}{\rightleftharpoons}}{\text { State of chest after operation. }}$ 
cause complete block and lead to collapse of the lung or infection. The tomograms showed no other abnormality.

Treatment.-Fruitless attempts at dilatation were made at intervals, till it was obvious that some other method of treatment would have to be tried.

The nature of the stenosis seemed quite evident : the only other possible alternative was congenita! agenesis of cartilage in the left main bronchus. I have seen such a case, but the lung was collapsed, and the history ruled out this diagnosis.

Excision of the stricture with repair, or failing that, as a last resort, pneumonectomy, seemed the only line of treatment likely to cure the disability.

Early in February, 1948, left thoracotomy was performed. The pleural cavity was quite free of adhesions. The left main bronchus was isolated and was fractured about its centre, the break in continuity of the cartilage being felt easily. There was no evidence of previous trauma to the mediastinum, except that the fracture line was firmly adherent to the pulmonary artery by fibrous adhesions. There was no suggestion of some other pathological process being present. The stricture was then resected, leaving only a narrow portion of membranous posterior wall of the bronchus joining the two ends; care was taken to preserve intact the bronchial artery going to the distal stump. End-to-end anastomosis of the two

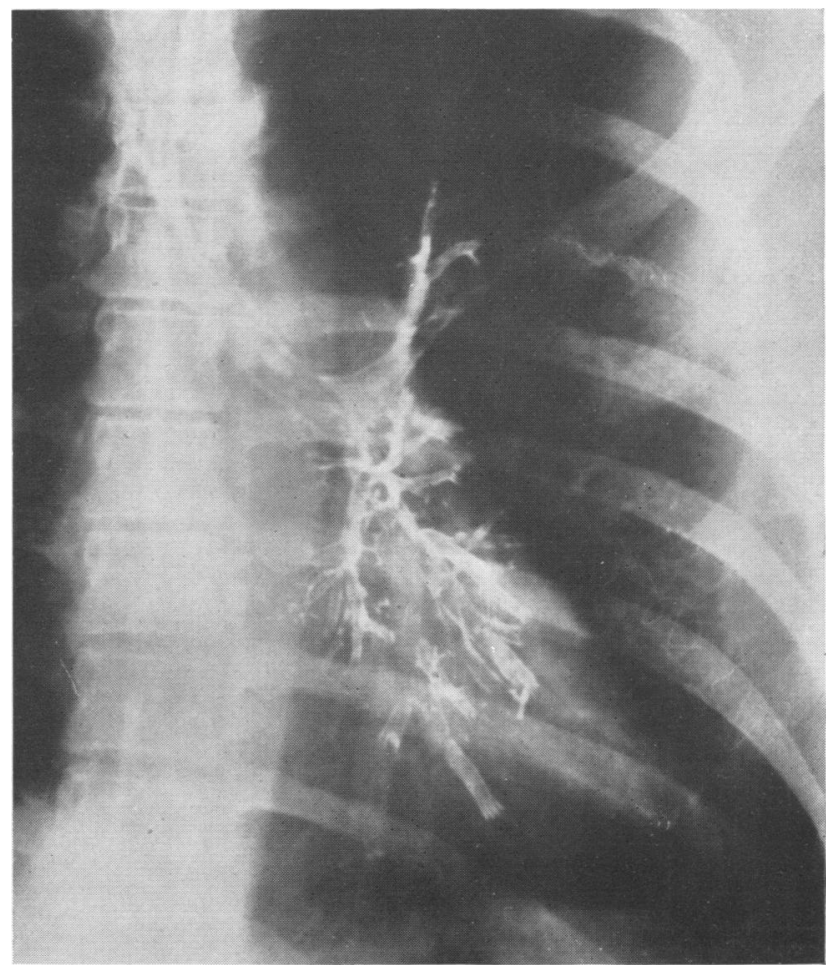

FIG. 6.-Radiograph (Aug 28, 1948) after instillation of lipiodol in left main bronchus showing absence of stenosis of left main bronchus after operation.

ends of severed broncilus was performed, using interrupted sutures going through all layers of the bronchial wall. It was necessary to be careful to take exactly equal bites on each side to prevent overlapping. No. 38 stainless steel wire was used to avoid as far as possible stricture formation at a later date and infection around the sutures. Fascial tissue from

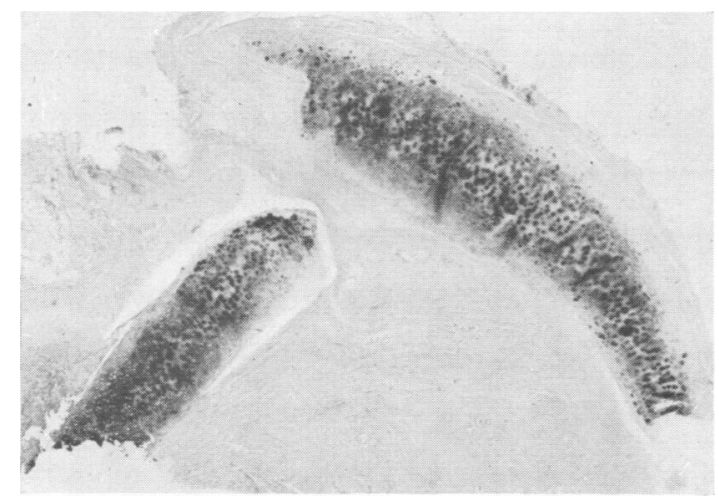

FIG. 5.-Photomicrograph of actual stricture of bronchus removed at operation. This shows point of fracture of cartilage of bronchial wall. the back wound was then wrapped round the join to reinforce and protect it. At the end of the operation positive pressure inflated the lung readily, and there was no leak of air through the suture line. An intercostal tube was inserted and connected with an underwater seal as a safety valve.

Convalescence was uneventful. No pneumothorax and no atelectasis occurred. The wheeze and audible rhonchus disappeared.

The pathological report on excised portions of bronchus stated:

"This certainly looks as though the cartilage had been fractured. There is a zone of rather vascular fibrous tissue between two pieces of cartilage whose apposing edges are very irregular."

The patient was discharged from hospital at the beginning of March, 1948.

Progress.-The patient has been examined at regular intervals. She has had no further chest troubles. Wheezing has not returned and there is no dyspnoea on moderate exercise. She has been working hard throughout the summer. Her only complaint is that she tires more quickly than formerly and has occasional dry cough.

Bronchoscopy was last performed in October, 1948. and revealed a normal left bronchial tree. The 
mucosa in the region of the anastomosis was in continuity, no ulceration being present, and the wire sutures were not visible. The only abnormality was the absence of normal motility in the region of the anastomosis.

In October, 1948, the patient's vital capacity was 2,700 ; before operation it was 2,100. A radiograph taken in October, 1948, showed some peaking of the left diaphragm, but otherwise there was no abnormality. Lipiodol was instilled to outline the left main bronchus; the radiograph showed no difference from the normal.

I am hopeful that a fibrous stricture will not develop.

\section{Discussion}

Kinsella and Johnsrud in 1947 published a comprehensive review of the literature on traumatic rupture of the bronchus, and recorded two cases of their own. They give details of some 38 cases reported since 1871 , when Winslow described rupture of a bronchus which occurred in a wild-duck.

In 1927 Krinitzki reported a case found by chance at autopsy examination. The right main bronchus terminated in a blind pouch $3 \mathrm{~cm}$. from the tracheal bifurcation and surrounded by fibrous tissue. About $3 \mathrm{~cm}$. beyond this there was a complete absence of bronchus, but distal to it the termination of the right bronchus was found with its proximal end obliterated by scar tissue entering a collapsed lung. There was a history of severe chest injury 21 years previously. The subject examined was the first case known of survival after traumatic rupture of a bronchus.

Recovery occurred in 19 of the cases reported, and in all of them permanent collapse of the lung resulted. The injury occurred most commonly in young men, although the youngest on record was three years old and the oldest seventy-four. In most of the cases severe chest injury of the compression or crush type had occurred, the result of being run over; less commonly damage resulted from a direct blow or fall. Rupture of the bronchus always occurred in the main bronchus, at the carina or within a few centimetres of it. Rupture was partial or complete. Kinsella and Johnsrud had one case in which the upper lobe bronchus was completely detached from the main stem, as well as rupture of the main bronchus $2.5 \mathrm{~cm}$. from the carina. The patient survived, and the precise nature of the injury was demonstrated by instillation of iodized oil.

The right and left main bronchi have been involved with equal frequency. Tearing of the lung parenchyma has not been a common association with this injury. The pulmonary artery and veins appear to have escaped injury in most of the reported cases. Following tearing of the bronchus, pneumothorax developed in 16 of the reported cases. This did not always progress to the tension type. Surgical emphysema occurred in 12 cases; it was dangerous and usually fatal when it resulted from bronchial rupture within the mediastinal pleura. Haemothorax occurred in 10 cases and bleeding into the lumen of the bronchus contributed to the production of atelectasis of the involved lung. Haemoptysis occurred sometimes. Rib fractures were found in about half of the recorded cases only.

Paul Sanger, in 1945, reported two cases of bronchial rupture due to penetrating wounds of the chest by shell fragments. These are the only two cases that I have been able to find in the literature in which laceration of the bronchus resulted from a penetrating injury.

Mechanism.-Rupture of the bronchus is not the result of penetration by fractured ribs, but appears to be due to a severe shearing force which results in the relatively rigid bronchus breaking, whereas softer structures, such as lung tissue proper and vessels, may escape damage.

Schönberg (1912) suggests that actual compression of the bronchus may occur between a relatively elastic chest wall anteriorly and rigid spine posteriorly, and that at the time of impact the lungs are in the expiratory phase with the glottis closed.

Treatment.-Kinsella and Johnsrud (1947) advise emergency thoracotomy with repair of the defect in the bronchus if the condition is diagnosed in the early stages. Bronchoscopy is obviously essential for exact diagnosis. Thoracotomy was performed by Sanger (1945) in the two cases mentioned previously. Tears between 2 and $2.5 \mathrm{~cm}$. in extent were present in the main bronchus in both patients, and both resulted from penetration of the chest by shell fragments. Sanger successfully repaired the tears by simple suture and applied a muscle graft around. Each patient did well ; the lung on the affected side re-expanded and no leakage of air occurred from the suture line. There is no record of any other case being treated in this way.

Treatment in the early stages has been expectant or palliative in the other cases reported.

Traumatic rupture of the bronchus with permanent collapse of the lung is compatible with life, provided the patient survives the immediate effects of the injury. In some of the reported cases attempts at dilatation of the resulting stricture of the bronchus have been made in order to 
bring about aeration of the collapsed lung, but have met with partial success in two cases only, reported by Piaget in 1939 and Löffler and Nager in 1941.

In the absence of infection in the collapsed lung, to relieve dyspnoea and to correct chest discomfort and displacement of the mediastinum, artificial pneumothorax has been performed by Clerf (1940), and also by Tyson and Lyle (1942). This treatment brought about relief of symptoms. Thoracoplasty has also been advocated to counteract the evil effect of excessive mediastinal shift.

Jones and Vinson (1939) advise pneumonectomy, should infection occur in the atelectatic lung. Pneumonectomy was performed in one case of this type by Kinsella (1947), but is the only one recorded to date.

\section{SUMmary}

An example of traumatic fracture of the left stem bronchus is described. The patient was a woman, aged 37, who was injured in a motor-car accident in July, 1947. In February, 1948, the condition was diagnosed and treated by excising the stricture, which had formed at the site of the fracture, and joining the cut ends of the bronchus by endto-end anastomosis. The patient made an uninterrupted convalescence, and the bronchial tree is now normal. The literature upon this topic is reviewed and discussed.

I would like to express my thanks to Dr. Woolmer, of Bristol Royal Infirmary, for solving the anaesthetic problem; I am grateful to Mr. Ronald Belsey for his encouragement over the surgical procedure, and to Professor T. A. Hewer, of Bristol University, for his pathological examination of the specimen and report.

\section{REFERENCES}

Clerf, L. H. (1940). Surgery, 7, 276.

Jones, F. W., and Vinson, P. P. (1939). Surgery, $5,228$.

Kinsella, T. J., and Johnsrud, L. W. (1947). J. thorac. Surg., 16, 571.

Krinitzki, S. I. (1927). Virchows Arch., 266, 815.

Löffler, W., and Nager, F. (1941). Schweiz. med. Wschr., 71, 293.

Piaget (1939). Ann. Oto-rino-laryn., 58, 917.

Sanger, P. W. (1945). Ann. Surg., 122, 147.

Schönberg, S. (1912). Berl. klin. Wschr., 49, 2218.

Tyson, M. Dawson, and Lyle, John S. (1942). New Engl.J. Med., 226, 192. 